\title{
Challenges in prediction of right ventricular failure among recipients of a left ventricular assist device
}

\author{
Saurabh Malhotra, MD, MPH, FASNC, a and Rami Doukky, MD, MSc, FASNC ${ }^{\mathrm{a}, \mathrm{b}}$ \\ a Division of Cardiology, Cook County Health, Chicago, IL \\ b Division of Cardiology, Rush University Medical College, Chicago, IL
}

Received Sep 11, 2019; accepted Sep 11, 2019

doi: $10.1007 / \mathrm{s} 12350-019-01913-7$

\section{See related article, pp. 303-308}

The right ventricle (RV), though often ignored, is of paramount importance as RV dysfunction portends poor prognosis among patients with heart failure (HF), specifically those with left ventricular assist device (LVAD). ${ }^{1}$ RV failure after LVAD implantation is a significant problem affecting nearly $20 \%$ of all LVAD recipients. ${ }^{2}$ When $\mathrm{RV}$ failure is severe enough to require biventricular support, the 1-year mortality is nearly $60 \% .^{3}$ Thus, predicting post-LVAD RV failure prior to device implantation is critical. Number of clinical, hemodynamic, and echocardiographic models seem to provide modest predictive value for post-LVAD RV failure. ${ }^{4}$ Assessing pre-LVAD RV ejection fraction seems to be an intuitive measure to assess RV performance and forecast RV failure after LVAD. Though echocardiography is widely available, accurate assessment of RV function remains challenging, largely due to the crescent-like shape of the RV which limits the complete visualization of the RV in any given view. Cardiac magnetic resonance (CMR) is an excellent technique for visualization and quantification of $\mathrm{RV}$ volumes and function. However, many patients with severe HF have implantable devices that limit the effectiveness of CMR. This is specifically true for those with ventricular assist devices, in whom CMR is contraindicated. On the other hand, radionuclide imaging is

Reprint requests: Rami Doukky, MD, MSc, FASNC, Division of Cardiology, Cook County Health, 1901 W. Harrison St., Suite 3620,

Chicago, IL 60612, USA; rami_doukky@rush.edu

J Nucl Cardiol 2021;28:309-10.

1071-3581/\$34.00

Copyright (C) 2019 American Society of Nuclear Cardiology. able to offset some of the limitations of echocardiography and CMR, and is often utilized for assessment of RV function among patients undergoing an LVAD implantation.

In this issue of the Journal, Patel et al, ${ }^{5}$ report the relationship between pre-LVAD RV function and occurrence of RV failure after LVAD implantation. The authors performed a retrospective study of 46 patients (83\% men; mean age, 55 years) in whom assessment of RV function prior to LVAD implantation was performed by first-pass radionuclide angiography (FPRNA). RV function was quantified by anterior planar FPRNA, while left ventricular volume and function were calculated by SPECT radionuclide angiography. Presence of RV failure post LVAD was determined from the review of medical records and was defined as inotropic requirement for $>14$ days post $L V A D$ or need for an RV assist device. The authors found that 8 out the 46 patients $(20 \%)$ developed RV failure after LVAD implantation, and these patients were older when compared to those who did not develop RV dysfunction (63 \pm 9 years vs $53 \pm 12$ years, $P=.03$ ). However, preLVAD RV ejection fraction was not different between patients who developed RV failure and those who did not $(41 \%$ vs $40 \%, P=.79)$. Moreover, none of the preLVAD RV parameters (ejection fraction, dilation and global hypokinesis) were predictive of RV dysfunction following LVAD. Measures of RV performance on invasive right heart catheterization did not correlate with RV ejection fraction on FPRNA and were also not predictive of RV dysfunction post LVAD.

FPRNA has been historically considered as the gold standard for assessing RV ejection fraction, especially in patients with LVAD. ${ }^{6}$ Since the radioisotope traverses the right heart chambers, followed by the pulmonary artery and then the lungs, RV boundaries can be determined with minimal overlap from surrounding structures and RV ejection fraction be accurately quantified. 
However, the technique is challenging to perform and the accuracy of the results is operator dependent. The limited effectiveness of FPRNA in quantifying RV function was reported in a study by Daou et al, wherein ejection fraction could not be determined in approximately $25 \%$ of the patients undergoing FPRNA. ${ }^{7}$ Unlike the left ventricle, the RV has a complex geometry, and planar function assessment from a single view is likely to provide an incomplete assessment of the true RV function. Additionally, volumetric assessments on FPRNA are qualitative and so is the determination of chamber size. This incomplete assessment of RV function by FPRNA could be one of the reasons for the lack of a significant relationship with post-LVAD RV failure in this study. Additionally, as opined by the authors, patients referred for pre-LVAD FPRNA are often perceived to be at high risk of RV dysfunction, and this clinical bias could mask the prognostic benefit of assessing RV function prior to LVAD. Furthermore, the baseline RV function was mildly reduced among these patients, and the value of routine FPRNA for prognosticating patients prior to LVAD will require a population with a wide range of $\mathrm{RV}$ ejection fraction to more clearly define its value in risk stratification.

While there is little doubt that RV function determines post-LVAD prognosis, we must rethink whether FPRNA remains a contemporaneous approach for assessment of RV function. Accuracy of RV function in such a situation may be less critical than reproducibility so one can confirm a real change in RV function before and after LVAD. In this regard, gated blood pool SPECT (GBPS) may confer some advantages. GBPS involves tomographic imaging of the cardiac changes, similar to gated perfusion SPECT, and can thus delineate the ventricular chambers better than FPRNA. GBPS allows for simultaneous assessment of biventricular function, for quantification of chamber volumes, as opposed to qualitative assessment by FPRNA. Additionally, the atrial counts can be excluded by direct visualization of counts in the short axis and horizontal long axis views, which are part of standard GBPS acquisition. GBPS has been shown to have modest correlation with FPRNA, and while some studies have reported excellent reproducibility of RV ejection fraction, ${ }^{8}$ other have shown this to be poor. ${ }^{9}$

In summary, identification of effective predictors of $\mathrm{RV}$ failure after LVAD is dearly needed. Larger clinical studies are needed to assess the prognostic value of FPRNA across a range of RV function and establish the reproducibility of GBPS for RV function and its application in LVAD population. Additional measures of RV function, such as RV phase analysis (akin to RV strain on echocardiography) which can be determined from routine FPRNA and GPBS studies, need to be evaluated as possible predictors of clinical outcomes in LVAD patients.

\section{Disclosure}

Dr. Malhotra serves on the speaker's bureau of Pfizer, Inc (New York, NY). Dr. Doukky has received a research grant from Astellas Pharma Global Development (Northbrook, IL).

\section{References}

1. Lampert BC, Teuteberg JJ. Right ventricular failure after left ventricular assist devices. J Heart Lung Transpl 2015;34:1123-30.

2. Kirklin JK, Naftel DC, Pagani FD, Kormos RL, Stevenson LW, Blume ED, et al. Seventh INTERMACS annual report: 15,000 patients and counting. J Heart Lung Transpl 2015;34:1495-504.

3. LaRue SJ, Raymer DS, Pierce BR, Nassif ME, Sparrow CT, Vader JM. Clinical outcomes associated with INTERMACS-defined right heart failure after left ventricular assist device implantation. J Heart Lung Transpl 2017;36:475-7.

4. Mohamedali B, Doukky R, Karavalos K, Avery E, Bhat G. Mean arterial pressure to central venous pressure ratio: A novel marker for right ventricular failure after left ventricular assist device placement. J Card Fail 2017;23:446-52.

5. Patel V, Tam M, Palardy M, Konerman M, Murthy V. Assessing the utility of pre-operative first pass radionuclide angiography to predict right ventricular failure post left ventricular assist device implantation. J Nucl Cardiol 2019.

6. Kjaer A, Lebech AM, Hesse B, Petersen CL. Right-sided cardiac function in healthy volunteers measured by first-pass radionuclide ventriculography and gated blood-pool SPECT: Comparison with cine MRI. Clin Physiol Funct Imaging 2005;25:344-9.

7. Daou D, Van Kriekinge SD, Coaguila C, Lebtahi R, Fourme T, Sitbon $\mathrm{O}$, et al. Automatic quantification of right ventricular function with gated blood pool SPECT. J Nucl Cardiol 2004;11:293-304.

8. Lairez O, Delmas C, Fournier P, Cassol E, Mejean S, Pascal P, et al. Feasibility and accuracy of gated blood pool SPECT equilibrium radionuclide ventriculography for the assessment of left and right ventricular volumes and function in patients with left ventricular assist devices. J Nucl Cardiol 2018;25:625-34.

9. Jensen MM, Haase C, Zerahn B. Interstudy repeatability of left and right ventricular volume estimations by serial-gated tomographic radionuclide angiographies using a cadmium-zinc-telluride detector gamma camera. Clin Physiol Funct Imaging 2015;35:418-24.

Publisher's Note Springer Nature remains neutral with regard to jurisdictional claims in published maps and institutional affiliations. 